\title{
Lung cancer drug therapy in Hungary - 3-year experience
}

This article was published in the following Dove Press journal:

OncoTargets and Therapy

13 May 2015

Number of times this article has been viewed

\author{
Judit Moldvay' \\ György Rokszin² \\ Zsolt Abonyi-Tóth ${ }^{2}$ \\ Lajos Katona ${ }^{3}$ \\ Katalin Fábián ${ }^{4}$ \\ Gábor Kovács ${ }^{5}$ \\ 'Department of Tumor Biology, \\ National Korányi Institute of \\ Pulmonology, Semmelweis University, \\ Budapest, Hungary; ${ }^{2} \mathrm{Rx}$ Target Company \\ Ltd, Szolnok, Hungary; ${ }^{3}$ Department \\ of Psychiatry and Psychotherapy, \\ Semmelweis University, Budapest, \\ Hungary; ${ }^{4}$ Department of Pulmonology, \\ Semmelweis University, Budapest, \\ Hungary; ${ }^{5}$ National Korányi Institute \\ of Pulmonology and Tuberculosis, \\ Budapest, Hungary
}

Correspondence: Katalin Fábián

Department of Tumor Biology, National Korányi Institute of Pulmonology, Semmelweis University, Diósárok I/c, Budapest II 25, Hungary

Tel +36303885443

Fax +36 I 2142498

Email drfabian.katalin@gmail.com
Abstract: Hungary is a world leader in lung cancer deaths, so it is of crucial importance that patients have access to modern treatments. The aim of our analysis was to explore how drug treatments are used in Hungary and how they are compatible with international practice. The inpatient and prescription database of the National Health Insurance Fund Administration of Hungary was used to study the frequency of certain chemotherapy protocols and duration of therapies during a 3-year period (2008-2010). During the study period, 12,326 lung cancer patients received first-line chemotherapy, a third of those $(n=3,791)$ received second-line treatment, and a third of the latter $(n=1,174)$ received third-line treatment. The average treatment duration was between 3 and 4 months. The first-line treatment of non-small-cell lung carcinoma mainly consisted of platinum treatment in combination with third-generation cytotoxic agents. A downward trend of gemcitabine, still the most common combination compound, was observed, in parallel with a significantly increased use of paclitaxel, and as a consequence carboplatin replaced cisplatin. Among the new agents, the use of pemetrexed and bevacizumab increased. Pemetrexed appeared mainly in second-line treatment, while erlotinib appeared also in second-line but mostly in third-line treatments. The first-line treatment of small-cell lung carcinoma consisted of a platinum-etoposide combination, while in the second-line setting topotecan was the most commonly used drug. According to our results, the chemotherapeutic combinations and sequencing are in accordance with international and national recommendations. Further detailed analysis of the available data may help to obtain a more accurate picture of the efficacy of lung cancer treatments as well.

Keywords: lung cancer, chemotherapy, molecularly targeted treatment, Hungarian practice

\section{Introduction}

In recent years, an impressive and fast development has been observed in drug therapy for lung cancer, a disease that still poses a challenge for clinicians. Thanks to modern molecularly targeted agents, long survival, which had never been achieved earlier, can be seen in some subtypes of the disease. However, in the treatment of advanced lung cancer, conventional cytotoxic chemotherapy still plays a crucial role, although a number of new molecularly targeted therapies have become available in routine clinical practice. These advanced treatments, however, are still only applied in about $10 \%-15 \%$ of lung cancer patients. The Korányi Bulletin (yearbook of the National Korányi Institute for Pulmonology and Tuberculosis), only includes data of the 6,000-6,500 new patients who are registered in the Hungarian pulmonary network. ${ }^{1}$ In contrast, the National Cancer Registry lists around 10,000-11,000 new lung cancer cases yearly; however, this cancer registry does not give information on the various 
therapeutic modalities. ${ }^{2}$ Although the pulmonary registry covers only $60 \%-70 \%$ of the total lung cancer incidence, it provides more detailed information, which can be extrapolated to our overall lung cancer population; therefore, we can realistically infer the national epidemiological data and treatment practices. Unfortunately, Hungary is the leader in lung cancer mortality in males in Europe (Figure 1). ${ }^{3}$ Moreover, in Central Europe our country tops lung cancer mortality statistics in both sexes.

According to the data of the National Korányi Institute, the most common type of lung cancer is adenocarcinoma, which surpassed in frequency squamous cell lung cancer during the last 10 years (Figure 2). In Hungary, an X-ray screening program for detecting tuberculosis has been running for decades, and due to this screening program $20 \%-25 \%$ of new lung cancer patients are diagnosed at earlier stages and with no symptoms. There are remarkable differences in stages at the time of diagnosis depending on the type of discovery (ie, during regular screening or after complaints) (Figure 3). During the last couple of years, about 40\%-50\% of newly diagnosed lung cancer patients received chemotherapy, and this proportion also depended on the type of lung cancer discovery (Figure 4). Of these patients, 20\% received chemotherapy perioperatively (ie, in a neoadjuvant or adjuvant setting), $20 \%$ in combination with irradiation, and about

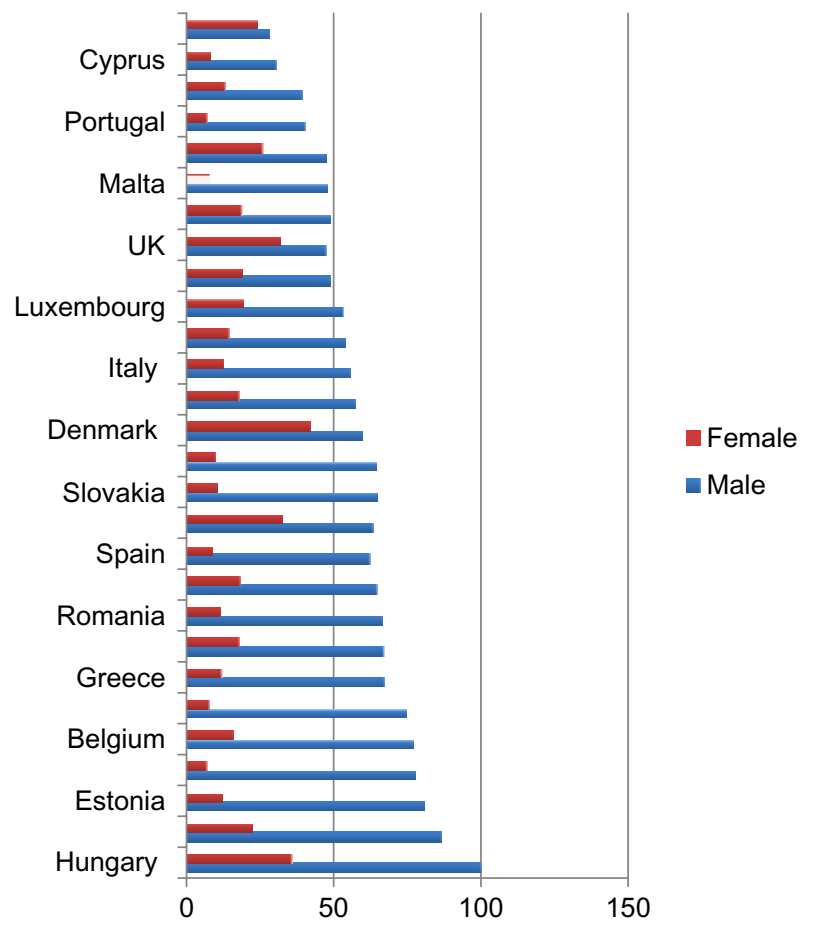

Figure I Lung cancer (ICD- 10 codes C33-C34), European age-standardized mortality rates, EU-27 countries, 2008 estimates.

Note: Adapted from Cancer Research UK, http://www.cancerresearchuk.org/ cancer-info/cancerstats/types/lung/mortalityl. Accessed January, 20I4.'

Abbreviation: ICD, International Classification of Diseases.
$60 \%$ received solely chemotherapy. Regarding molecularly targeted treatment, national data collection has not been done yet. However, thanks to item-based funding data, the National Health Insurance Fund Administration will have detailed information about these treatments soon.

\section{Materials and methods}

In order to gather additional information regarding the drug therapy of Hungarian lung cancer patients, we performed an analysis using the outpatient and inpatient care data, as well as that obtained from the prescription database of the National Health Insurance Fund Institutional Repository with reference to $\mathrm{C} 33 \mathrm{H} 0-\mathrm{C} 3490$ International Classification of Diseases codes (National Health Insurance Fund Administration license 44-P-307/2011). ${ }^{4}$ The purpose of the study was to determine the therapeutic sequences and the application frequency of active ingredients used, as well as the development of drug combinations between January 1, 2008 and December 31, 2010. The research was carried out using a mathematical algorithm developed by RxTarget.

The determination of therapeutic sequences included only the patients, who received no chemotherapy in 2007 and did not pick up oncologic prescriptions at public pharmacies. In their cases, it was possible to determine clearly what the first type of treatment was. However, information was not available on whether the first applied drug therapy was the real first-line treatment or the patient had already been treated for lung cancer prior to 2007. Likewise, it was not possible to distinguish adjuvant chemotherapy from treatment during disease progression or palliative drug therapy; only the succession of treatments could be analyzed. Our results show that the distribution of first-, second-, and third-used drug therapy was largely in line with the expected distribution of first-, second-, and third-line treatments. We regarded a treatment as a new line when a new chemotherapy regimen was applied, or if under the same protocol the patient picked up an oncologic prescription that had not been picked up 90 days prior to or in combination with other chemotherapy more than 30 days after the onset of the first cycle, or at any time after the onset of the second cycle. The break to be held at the end of therapeutic protocols was taken into account when we determined the durations of therapies. When we examined the proportion of individual therapies, patients treated in 2007 were also taken into account, because in their cases it was not necessary to know the location of the current treatment within the therapeutic sequence. We reviewed the available data and summarized - and in some cases also plotted - each treatment sequence and each major product. 


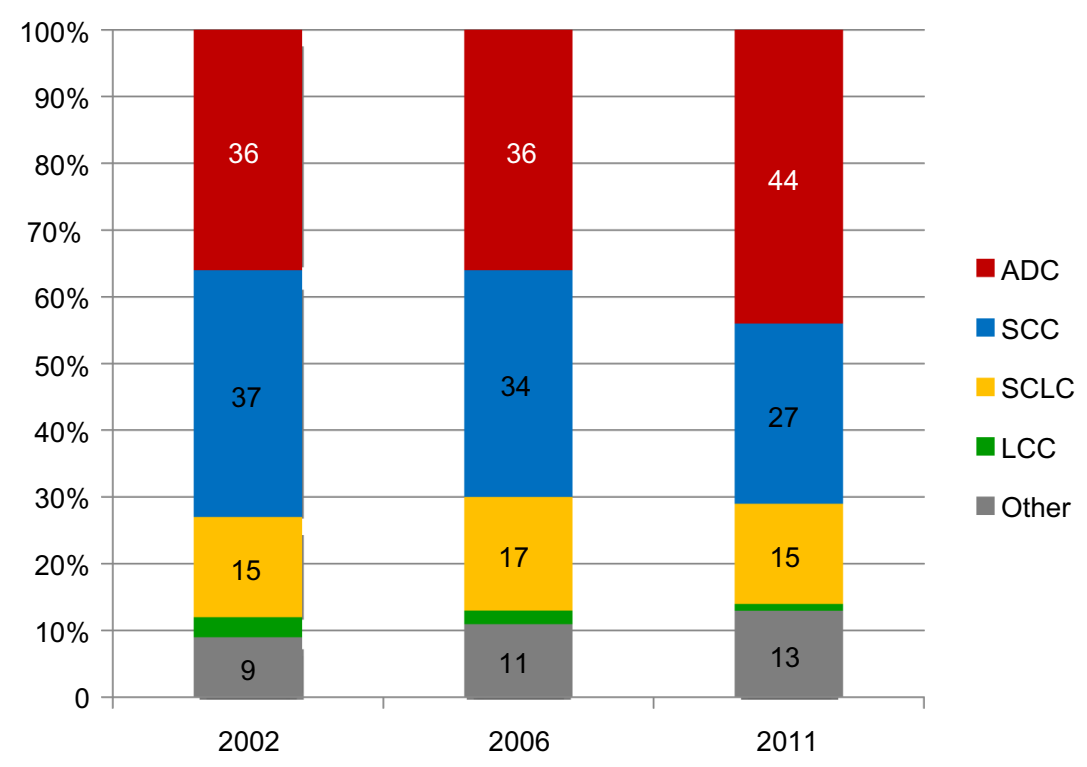

Figure 2 Histologic distribution of lung cancer in 2002 ( $n=6,361), 2006(n=6,519)$, and 201 I $(n=6,205)$.

Abbreviations: ADC, adenocarcinoma; SCC, squamous cell carcinoma; SCLC, small-cell lung cancer; LCC, large-cell carcinoma.

When we examined the development of drug combinations, linear regression was used to determine changes in patient numbers over time.

\section{Results}

\section{Treatment sequences}

The number of patients who received a variety of lung cancer therapies during the 3-year period is shown in Figure 5. It can be seen that less than a third of patients receiving first-line treatment received second-line therapy, and also less than a third of patients receiving second-line treatment (ie, less than
$10 \%$ of patients with first-line treatment) received third-line therapy. Fourth-, fifth-, and sixth-line treatments were applied in only a small number of cases; therefore, particular conclusions could not be drawn in those cases.

Nowadays, the standard first-line treatment in lung cancer is platinum-based combination cytotoxic chemotherapy; this is reflected in the case of treatments received for the first time as well. If we look at other drugs in the combinations, it can be seen that the most often used drug was gemcitabine, and the median duration of treatment varied between 3 and 4 months. This demonstrates that national recommendations

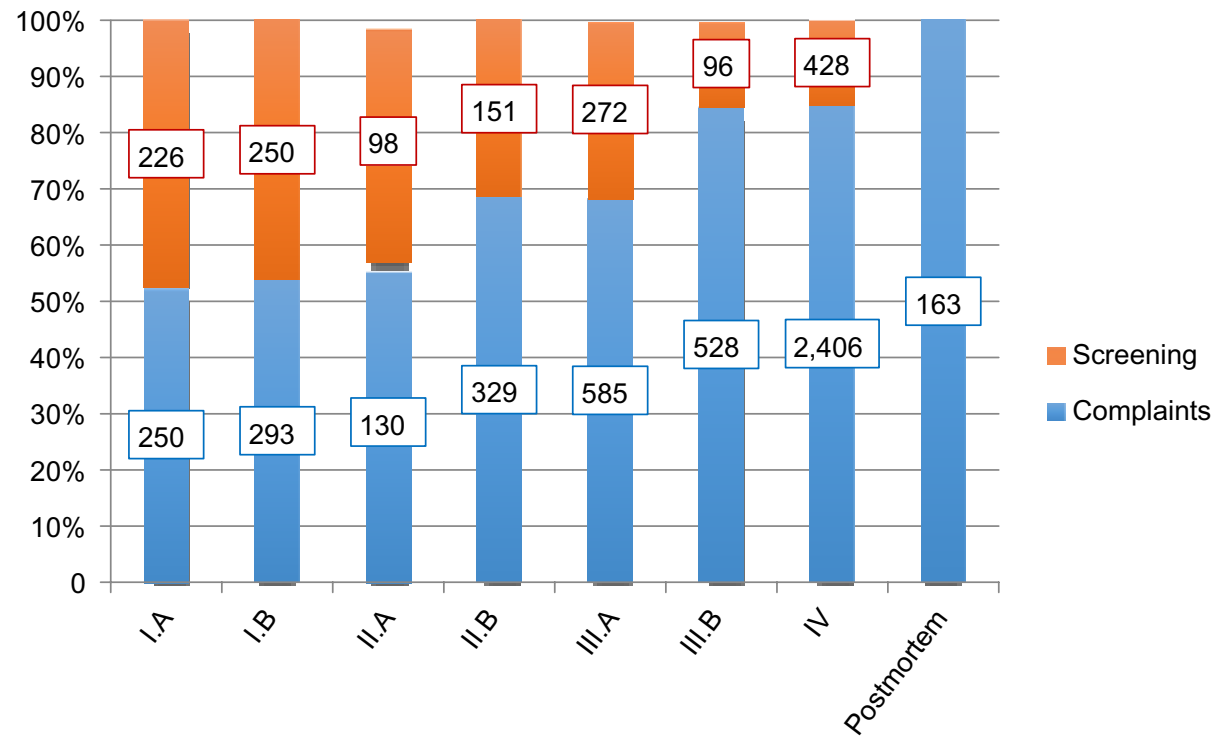

Figure 3 Stages of lung cancer at the time of diagnosis by the type of discovery $(20 \mathrm{Il}, \mathrm{n}=6,205)$. 
A

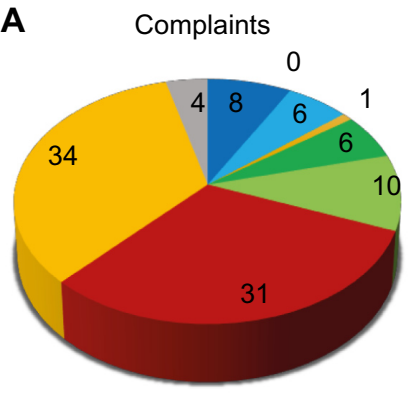

- Surgery

- Neoadjuvant + surgery

- Surgery + adjuvant

- Surgery +

chemoradiotherapy

- Radiotherapy

- Chemoradiotherapy

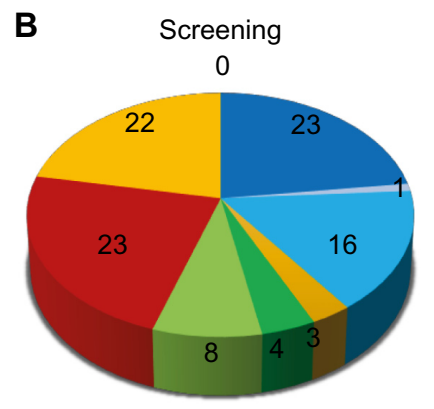

- Surgery

- Neoadjuvant + surgery

- Surgery + adjuvant

॥ Surgery + chemoradiotherapy

- Radiotherapy

- Chemoradiotherapy

Figure 4 Therapeutic modalities (\%) in new lung cancer cases by the type of discovery. Notes: Type of discovery: (A) complaints, (B) screening (2011, n=6,205).

are in line with international recommendations, which are satisfied by the application of four treatment cycles. The higher median number of platinum-etoposide combinations results from the fact that for small-cell lung cancer, both the national recommendations and reimbursement allow the use of six cycles. Special mention should be made of the 85 patients who received bevacizumab-carboplatin-paclitaxel as a first-line treatment. In their cases, the median length of therapy was 3 months. It should be noted, however, that this figure does not include additional cycles of bevacizumab monotherapy used as part of the protocol. A similar figure -
87 patients - was treated first with radiation therapy combined with cisplatin and docetaxel. From the less frequently used combinations, carboplatin-docetaxel (109 patients, median length 3 months), cisplatin-pemetrexed (106 patients, median length 3 months), cisplatin-vinorelbine, often as adjuvant therapy (77 patients, median length 2 months), and cyclophosphamide-epirubicin-vincristine for the treatment of small-cell lung cancer (138 patients, median length 4 months) should be emphasized. The surprisingly low median length of adjuvant treatments suggests that professionals often stop these treatments even in the case of milder toxicity.

The composition of second therapies complies with recommendations for second-line treatment, as the three drugs registered for second-line treatment of non-small-cell lung cancers rank at the top. Among them, pemetrexed is the most frequently used drug, followed by docetaxel and then the nowadays most commonly used molecularly targeted treatment: the EGFR tyrosine-kinase inhibitor erlotinib with a median length of therapy of 4 months.

In the case of third-line therapy, erlotinib, the well-tolerated and in our country mostly applied drug in K-ras mutation-negative adenocarcinomas, leads. The median length of therapy is 4 months, indicating the legitimacy of the applied negative selection method.

\section{Development of drug combinations in non-small-cell lung cancer}

Figure 6 is an overview of non-small-cell lung cancer treatments applied between January 1, 2008 and December 31, 2010. Figures show 1) changes in the number of patients treated, 2) the proportion of patients treated with certain drugs compared to the total number of patients, and 3) the

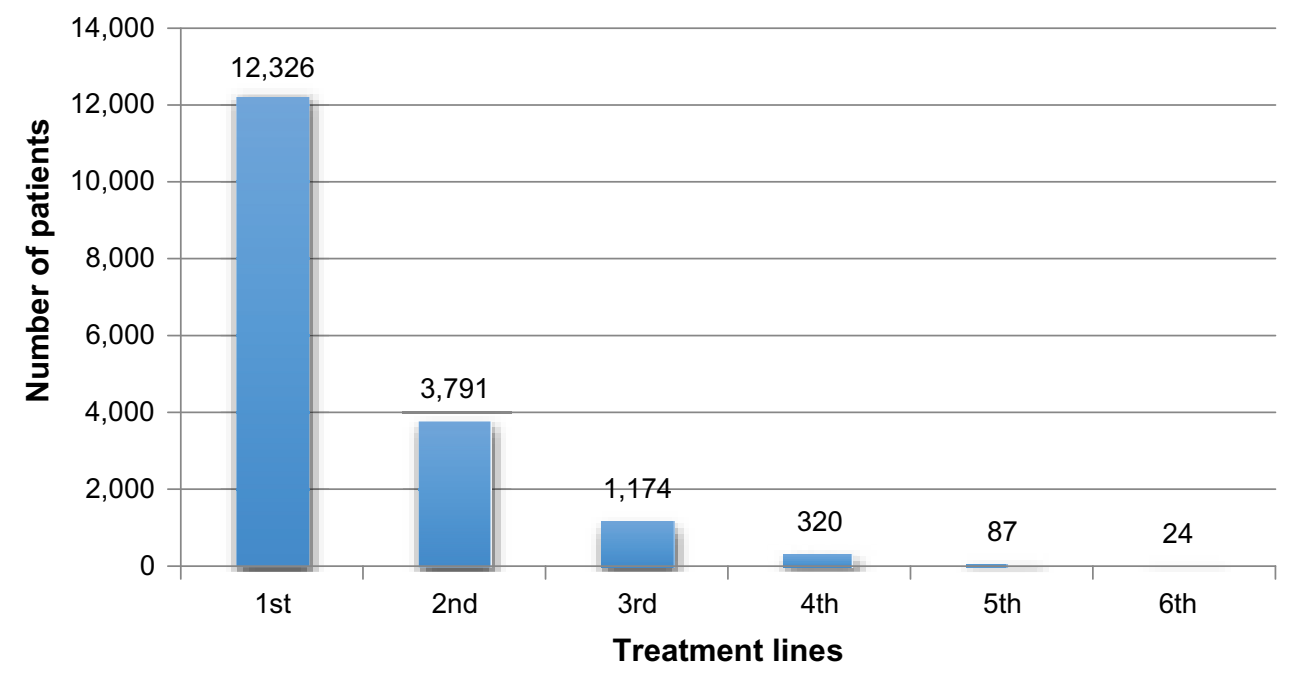

Figure 5 Lung cancer: number of patients receiving different therapies (January I, 2008 to December 2I, 20I0.) 


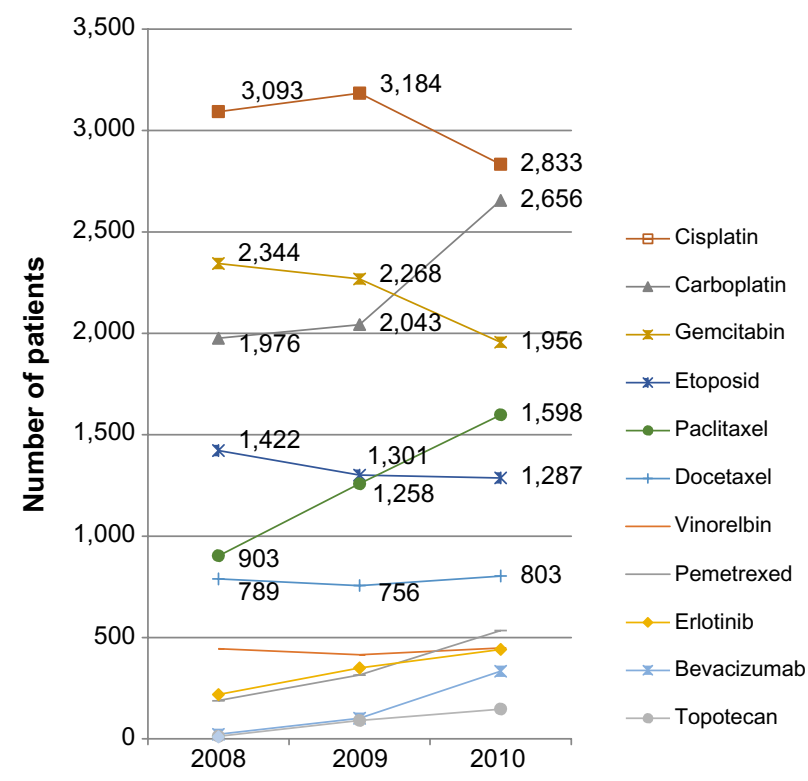

Figure 6 Lung cancer: number of patients who received chemotherapy and/or drug therapy in any treatment lines.

proportion of patients treated with certain substances in each treatment step (first, second, and subsequent therapy) compared to the total number of patients treated in that particular therapeutic step. The individuals who were in a phase of chemotherapy beginning in the same month or who picked up oncologic prescription at a pharmacy were regarded as treated patients. Where the number of patients allowed it, monthly statistics were used. Where this was not possible, data are presented by year. In the latter case, regression analysis was not performed. We considered a therapy "old" when treatments started before 2008 .
Upon examination of the evolution of the annual number of patients, it can be seen that the leading role of cisplatin is decreasing in parallel with an increase in the number of patients receiving carboplatin. This correlates with the decreasing tendency of gemcitabine administration and the increased application of paclitaxel. Detailed data analysis shows that the absolute number with patients on cisplatin $(P=0.018)$, gemcitabine $(P<0.001)$, and etoposide $(P=0.012)$ treatment is reduced. The number of patients receiving carboplatin, paclitaxel, and new-therapy products of recent years, such as pemetrexed, erlotinib, bevacizumab, and topotecan $(P<0.001)$, is increasing. Docetaxel and vinorelbine $(P=0.7)$ are stagnating.

According to data analysis of patients on first-line treatment, the proportion of cisplatin, gemcitabine $(P<0.001)$, etoposide $(P=0.041)$, docetaxel $(P=0.004)$, and vinorelbine $(P=0.003)$ decreased. In contrast, the proportion of carboplatin, paclitaxel, bevacizumab $(P<0.001)$, and pemetrexed $(P=0.009)$ is increasing. Erlotinib is stagnating $(P=0.415)$. In the case of carboplatin-paclitaxel versus cisplatin-gemcitabine, a marked change can be observed in September 2009 (Figure 7).

When we look at the agents that are most frequently used as a second-line treatment, the following can be observed: the proportion of docetaxel - apart from cycles that started before $2008(P=0.737)$ - decreased significantly (first, $P=0.004$; second and third, $P<0.001$ ). Its role as a first-line therapy is insignificant compared to second- and third-line therapy (Figure 8).

The proportion of pemetrexed as both first- and second-line treatment is on the rise (first, $P=0.009$; second,

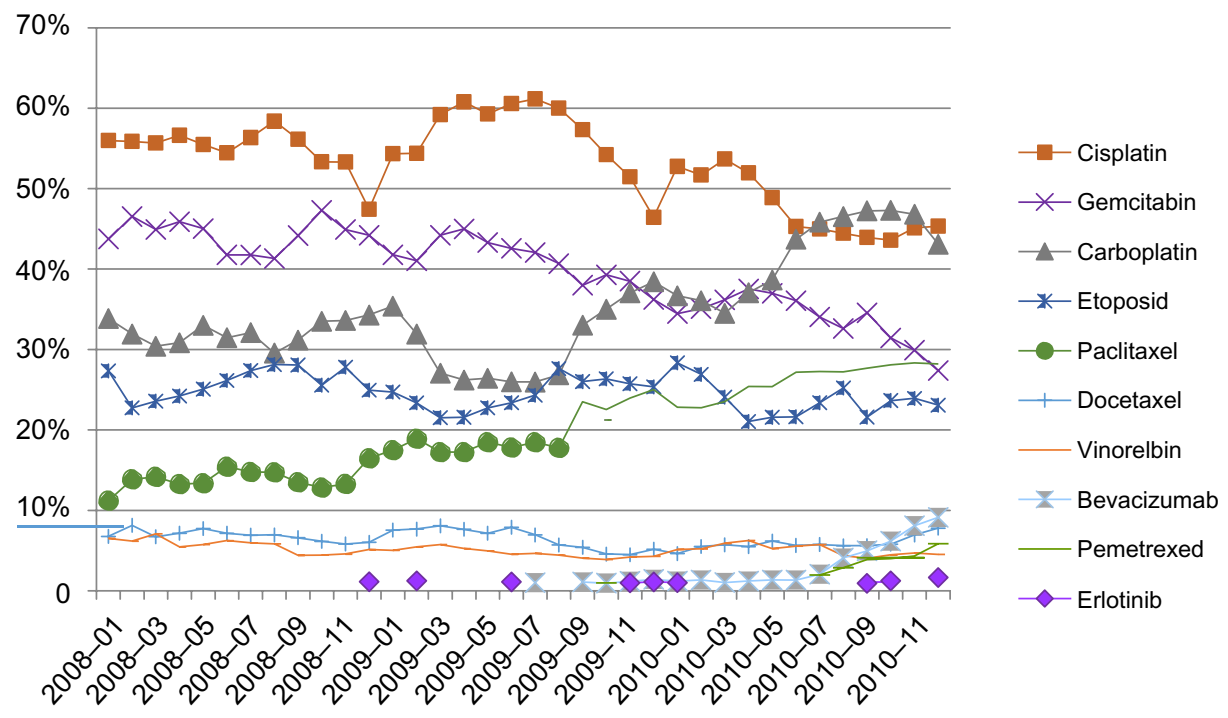

Figure 7 Lung cancer: percentage of patients receiving first-line treatment. 


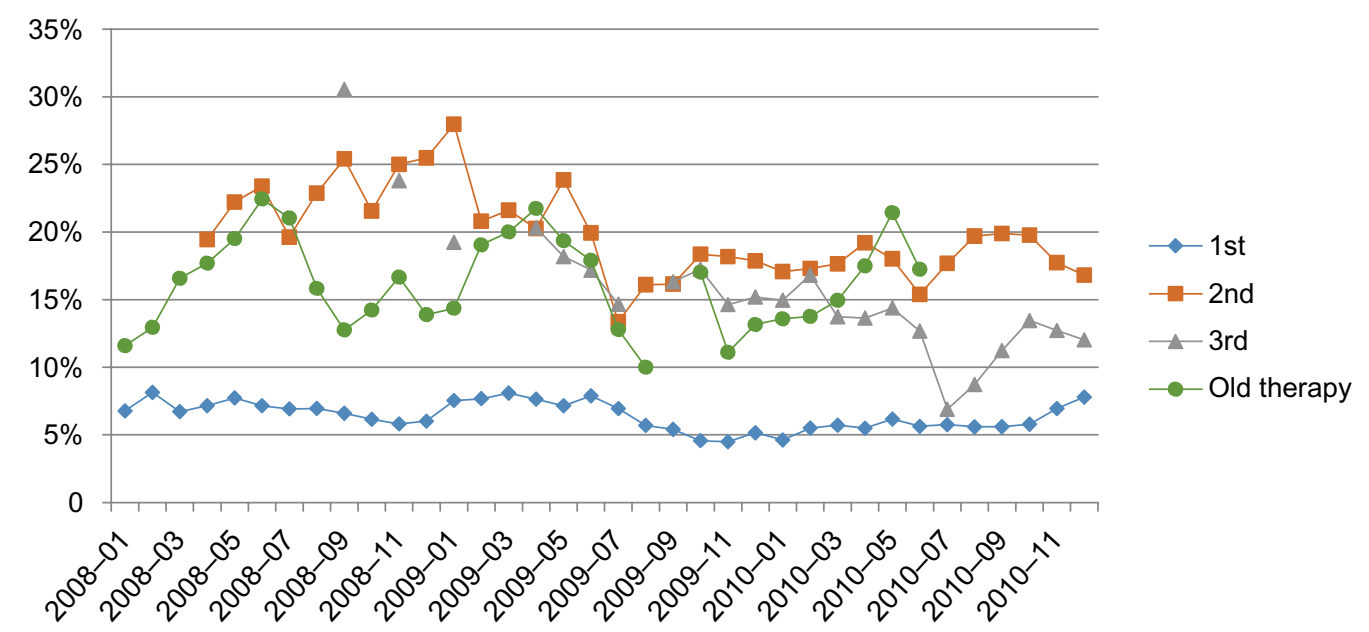

Figure 8 Lung cancer: percentage of patients treated with docetaxel in different therapy lines.

Note: We considered a therapy “old" when treatments started before 2008.

$P<0.001$; pre-2008, $P=0.002$ ) (Figure 9). It should be noted, however, that pemetrexed should not be used in third-line treatment. It is surprising, therefore, that in some cases it appears as a third-line treatment.

The use of erlotinib in first-line treatment is negligible (previously, it was only available as a study drug); however, its role in other therapeutic lines continues to grow (second, prior to 2008, $P<0.001$; third, $P=0.002$ ) (Figure 10).

\section{Discussion}

In Hungary, indications, compositions, and sequences of lung cancer treatment are regulated by a national protocol based on international guidelines. ${ }^{5-7}$ According to our detailed data analysis, the chemotherapy combinations and the order of their application are in line with international practice. The new products introduced in recent years (pemetrexed, erlotinib, and bevacizumab) have found their place in the oncology palette. In the case of molecularly targeted therapies (erlotinib and gefitinib), however, current Hungarian regulations - ie, patients having access to these molecular biological treatments under strict indication criteria and only in certain oncologic centers - make it difficult to access these medications, which raises fears that some patients miss out on this therapeutic modality that often helps achieve spectacular clinical and radiological regression and significant survival benefit.

Conventional cytotoxic chemotherapy still provides the basis of drug treatment of lung cancer, and platinum-based cytotoxic chemotherapy is the standard for first-line treatment. The most common combination drugs are gemcitabine, paclitaxel, and vinorelbine, and more recently pemetrexed. However, the choice is often "empirical"; decisions are

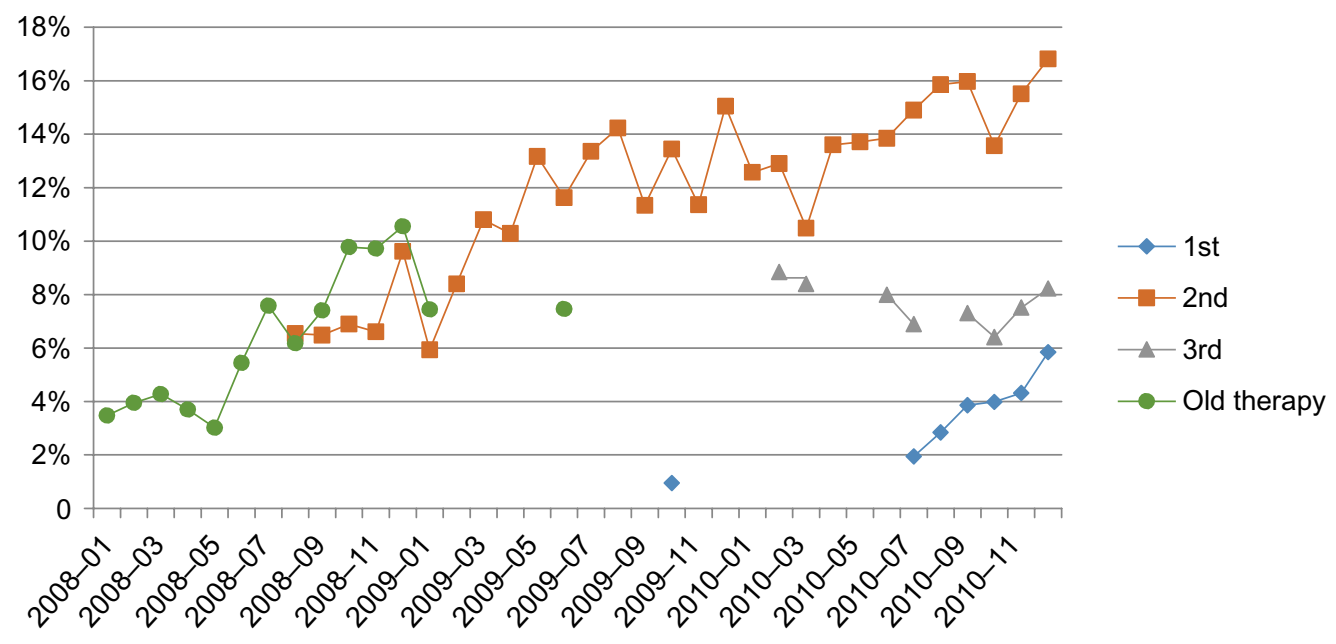

Figure 9 Lung cancer: percentage of patients treated with pemetrexed in different therapy lines. Note: We considered a therapy "old" when treatments started before 2008. 


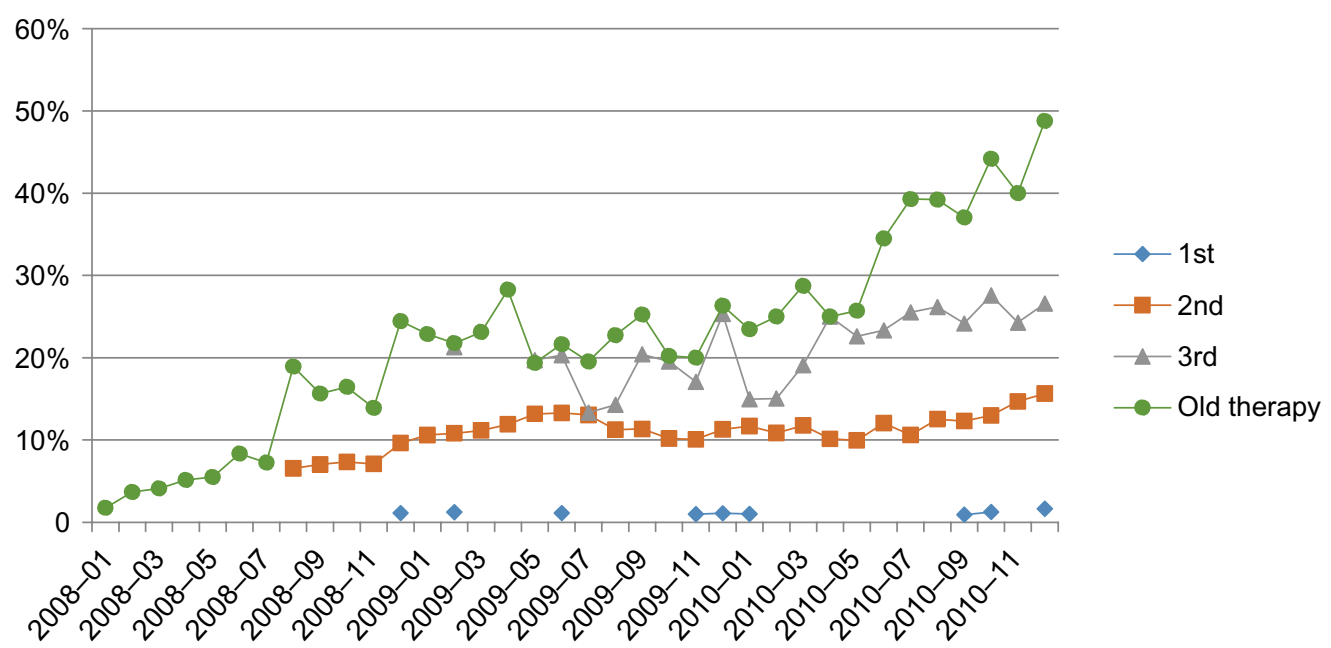

Figure 10 Lung cancer: percentage of patients treated with erlotinib in different therapy lines.

Note: We considered a therapy "old" when treatments started before 2008.

most commonly made on the basis of accessibility and the side-effect profile. In routine clinical practice, predictive tissue markers are not used for patient selection yet; however, a growing number of research results demonstrate that cytotoxic chemotherapy can also be tailored to individual cases. ${ }^{8}$ Personalized therapy is not only beneficial for the patient but can also make cancer treatment more effective and more efficient.

With the help of comprehensive data analysis, similar to that aforementioned, realistic pictures can be obtained about details and changes over time of treatments of a specific disease group. For example, it is noteworthy that analysis of such data in some cases revealed that patients were given a chemotherapeutic combination as an umpteenth - and perhaps even unnecessary - treatment, which neither oncologic evidence nor the performance status of the patient would have allowed. The lower median treatment lengths also support this presumption. While we most firmly have to stand up for the principle that patients with appropriate performance status and histological and molecular parameters have wide access to modern drugs providing them a substantial survival benefit, treatments giving mainly discomfort to the patient rather than providing a realistic chance of survival should not be forced. The nil nocere principle should not be forgotten in these treatments either. Overall, it should be emphasized that all the products that demonstrate therapeutic efficacy of high-level evidence are available for Hungarian physicians who treat lung cancer patients. Surely, this is one of the reasons lung cancer patient-survival probability was found to be the best in Hungary when compared with the results from Canada, Finland, Italy, and Norway, according to a data analysis in $2008 .^{9}$
The decreasing median length of therapy with cytotoxic agents compared with previous therapy lines might be explained by increased resistance, cumulative toxicity, and the assumption that patients' performance status was determined improperly. It might happen that physicians - with the best of intentions - set an unrealistically better performance status of the patient. That is one of the reasons we must strive to be able to determine this important factor in a more objective manner based on clear criteria. It is surprising that in some cases, platinum-based combination is used as an umpteenthline treatment, although some of these cases may represent umpteenth-line small-cell-lung cancer therapy. In non-smallcell lung cancer, these heroic treatments often do not make sense, but can "reassure" the physician and the patient that something is still happening. In such cases, the oncology team should take more responsibility when making decisions.

There are many other challenges in oncopulmonology we have to face. The expansion of molecularly targeted therapy enhances the role of other professionals - ie, pathologists and molecular biologists - who are crucial in patient selection. In addition, a change in attitude is needed among pulmonologists and bronchologists regarding the way of getting samples, the size of biopsy material, the number of biopsy pieces, the proportion of histological samples (versus cytological samples), and also the issues of rebiopsy.

This data analysis has several limitations. Neither neoadjuvant, adjuvant treatments, nor therapies used at disease progression were analyzed separately. When we determined median length of treatment, we could not use number of cycles as used in clinical practice, but measured started months, and they were not always identical. Moreover, treatments using study drugs were not included. Nevertheless, 
this work contains precious and valuable data that can and will be used in the analysis of item-based therapies. The purpose of the present study could not be the analysis of each modality of lung cancer management; however, in the future it would be worth analyzing comprehensively the opportunities of surgery and radiotherapy as well. Certainly, national imbalances in both personnel and material conditions and also in access to therapies could be revealed, but this could contribute to the initiation of changes aimed at faster and more efficient and effective management of patients.

If similar analyses in other oncology fields also become available, comparison of data could provide valuable information regarding quality assurance and cost-effectiveness. These can be of great importance, and can help Hungarian lung cancer specialists to meet future Hungarian and European challenges. ${ }^{10}$

\section{Acknowledgment}

This manuscript is an expanded English-language version of an article titled "Analysis of drug therapy of lung cancer in Hungary", published in Hungarian in Magyar Onkológia (2013), with the permission of the editor-inchief. During the study period, Lajos Katona worked as head of statistics and analysis for the National Health Insurance Fund Administration of Hungary. The study was funded by the National Korányi Institute of Pulmonology and Tuberculosis.

\section{Disclosure}

The authors report no conflicts of interest in this work.

\section{References}

1. Cancer Research UK. Lung cancer mortality statistics. 2014. Available from: http://www.cancerresearchuk.org/cancer-info/cancerstats/types/ lung/mortality. Accessed January 3, 2014.

2. Csoma Z, Kovács G, Ostoros G, et al. [Pulmonary institutions 2011 epidemiological and operational data]. Hungarian. 2012. Available from: http://www.koranyi.hu/tartalom/bulletin/Evkonyv2012.pdf. Accessed September 26, 2014.

3. Hungarian National Cancer Registry [homepage on the Internet]. Available from: http://www.oncol.hu/rakreg/stat2/nrr_2010.html. Accessed September 26, 2014.

4. RxTarget. [Oncology analysis - lung cancer treatment order]. 2011. Hungarian.

5. Ostoros G, Bajcsay A, Balikó Z, et al. [Lung cancer prevention, diagnosis and treatment principles]. Magy Onkol. 2012;56(2):114-132. Hungarian.

6. National Comprehensive Cancer Network. Non-small-cell lung cancer. Available from: http://www.nccn.org/professionals/physician_gls/pdf/ nscl.pdf. Accessed September 26, 2014.

7. National Comprehensive Cancer Network. Small-cell lung cancer. Available from: http://www.nccn.org/professionals/physician_gls/pdf/ sclc.pdf. Accessed September 26, 2014.

8. Simon GR, Schell MJ, Begum M, et al. Preliminary indication of survival benefit from ERCC1 and RRM1-tailored chemotherapy in patients with advanced non-small cell lung cancer: evidence from an individual patient analysis. Cancer. 2012;118(9):2525-2531.

9. Tusnády G, Gaudi I, Rejto L, Kásler M, Szentirmay Z. [Survival chances of Hungarian cancer patients in the National Cancer Registry]. Magy Onkol. 2008;52(4):339-349. Hungarian.

10. Kásler M, Ottó S. [European and Hungarian national tasks in oncology]. Magy Onkol. 2008;52(1):21-33. Hungarian.
OncoTargets and Therapy

\section{Publish your work in this journal}

OncoTargets and Therapy is an international, peer-reviewed, open access journal focusing on the pathological basis of all cancers, potential targets for therapy and treatment protocols employed to improve the management of cancer patients. The journal also focuses on the impact of management programs and new therapeutic agents and protocols on

\section{Dovepress}

patient perspectives such as quality of life, adherence and satisfaction. The manuscript management system is completely online and includes a very quick and fair peer-review system, which is all easy to use. Visit http://www.dovepress.com/testimonials.php to read real quotes from published authors. 